\title{
EFFICIENCY OF CYANOBACTERIAL INOCULATION OF WHEAT SEEDS TO REDUCE THE PHYTOTOXIC EFFECT OF GLYPHOSAT
}

\author{
Koval E.V. ${ }^{1}$, Ogorodnikova S. Yu. ${ }^{2}$ \\ ${ }^{l}$ Vyatka state university, ${ }^{2}$ Institute of Biology, Komi Scientific Center, Ural Branch of the Russian \\ Academy of Sciences, Russia \\ e-mail: undina2-10@yandex.ru
}

Glyphosate is one of the most popular pesticides on the modern market, but it is highly toxic to many plants, is absorbed by the leaves and moves to the roots, suppressing the vital activity of plants for a long time. Currently, the development of methods for reducing the phytotoxicity of contaminated substrates using microbial cultures is relevant.

The aim of the work was to study the effect of inoculation of wheat seeds with the cyanobacterium Nostoc paludosum Kutz. on glyphosate toxicity for plants.

Seeds of wheat (sort Iren) were germinated for a week with cyanobacteria and without them. Subsequently, the seedlings were transplanted to a solution of glyphosate $\left(1 \cdot 10^{-4} \mathrm{~mol} / \mathrm{l}\right)$ prepared on a Knop nutrient solution. In the phase of two leaves, the intensity of lipid peroxidation (LPO) processes in wheat roots and leaves, the accumulation of plastid pigments, substances with antioxidant properties, ascorbic acid and anthocyanins, and the length of seedling organs were evaluated. The age of cyanobacteria culture is 1.5 months, the titer is $6.5 \cdot 10^{7}$ cells $/ \mathrm{ml}$.

It was established that pre-sowing inoculation of seeds with cyanobacterium $N$. paludosum activated LPO processes in wheat leaves at $17 \%$ of the control level, which was determined by the accumulation of malonic dialdehyde - the main LPO reactions product. But its amount in the roots in this variant was extremely small (no more than $10 \%$ of the control). Significant changes in the content of plastid pigments (chlorophylls and carotenoids), anthocyanins, and ascorbic acid in the leaves of plants grown with cyanobacterial inoculation of seeds were not detected. In addition, cyanobacteria favorably influenced the growth of wheat roots, which exceeded control by $30 \%$.

Glyphosate had a toxic effect on wheat. The intensity of lipid peroxidation processes in leaves increased by 2.3 times compared with the control level. The content of anthocyanins and ascorbic acid, substances with antioxidant properties, increased in leaves by $90 \%$ and $50 \%$ times the control level, respectively. Their increased number indicates the activation of antioxidant protection in plant cells and is aimed at adapting plants to the action of the pesticide. The content of plastid pigments, in contrast, decreased. The toxic effect of glyphosate was also reflected in the length of the wheat organs, the length of the roots was lower than the control by $30 \%$, and the shoots - by $10 \%$.

Inoculation of seeds with cyanobacterium $N$. paludosum had a protective effect on plants that were grown in the presence of glyphosate. There was a decrease in the intensity of LPO processes in the roots and leaves of wheat grown using cyanobacteria on the glyphosate solution compared to the action of glyphosate without treatment with cyanobacteria. Under conditions of contamination of the growing environment with glyphosate, inoculation of seeds with cyanobacteria contributed to the accumulation of anthocyanins and ascorbic acid in wheat leaves, but to a lesser extent than without inoculation. A hign correlation was found between the content of malonic dialdehyde in wheat leaves and the amount of ascorbic acid $(\mathrm{r}=0.87)$. An increase in the content of chlorophylls in the leaves of plants that were treated with $N$. paludosum was noted, compared with plants that were grown on a substrate contaminated with glyphosate without inoculation. The length of the experimental plants treated with cyanobacteria and grown on glyphosate solution was higher than in the variant without cyanobacterial inoculation and returned to the control value.

Thus, inoculation of seeds with cyanobacterium $N$. paludosum had a phytoprotective effect on wheat plants in a glyphosate pollution. Cyanobacterial inoculation of seeds reduced the intensity of POL processes in leaves and roots of plants, caused an increase in chlorophyll content, stimulated the efficiency of the antioxidant system. As a result of biochemical rearrangements, linear growth is activated in plant inoculated with cyanobacteria. 